\title{
Drugging protein kinases in cancer: from small molecules to nanoparticles
}

\section{Introduction}

Protein kinases are a large family of enzymes that catalyze the transfer of a phosphate group from adenosine triphosphate (ATP) to the hydroxyl $(\mathrm{OH})$ group of an amino acid side chain in proteins. Most protein kinases act on both serine and threonine residues or on tyrosine residues in a protein. A few of them called dual-specificity kinases catalyze the transfer of phosphate group to all three residues. ${ }^{1}$ It has been estimated that approximately 500 protein kinases are encoded within the human genome; which represents approximately $1.7 \%$ of all human genes. ${ }^{2}$ Protein kinases regulate a wide array of cellular processes including cell division, metabolism, differentiation, motility, protein translation, protein turnover, cellular signaling and apoptosis. ${ }^{3}$ Often times they regulate cellular processes through a highly controlled series of phosphorylation reactions wherein activity of a downstream protein kinase is regulated by phosphorylation by another upstream protein kinase. Deregulated kinase activity is a frequent cause of disease, particularly cancer, where they regulate many aspects important in tumor progression and metastasis. In several instances protein kinases regulates cancer progression by phosphorylating ontogenesis and tumor suppressor proteins thereby regulating the activity, stability, and function. ${ }^{4-8}$ Interestingly, many of the tumor suppressor genes and dominant oncogenes identified so far are also protein kinases. Kinases such as c-Src, c-Abl, mitogen activated protein (MAP) kinase, phosphotidylinositol-3-kinase (PI3K) AKT, and the epidermal growth factor (EGF) receptor are commonly activated in cancer cells, and are known to contribute to tumor genesis. ${ }^{9-13}$ Given their importance in human diseases such as cancer, protein kinases have emerged as attractive therapeutic targets.

\section{Small molecule inhibitors and antibodies}

Several strategies have been developed to target protein kinases. Most popular among them is the use of small molecules or antibodies that block kinase-substrate interaction or those that bind the ATPbinding site of the enzyme inhibit its ability to drive catalysis. Several notable protein kinase inhibitors such as the Abl kinase inhibitor immatinib (gleevac), which is being used as afront-line cancer therapeutic for chronic myelogenousleukaemia and acute lymphocytic leukaemia, are active site inhibitors. ${ }^{14}$ Other examples include trastuzumab (HER2 inhibitor for treatment of breast cancer), bevacizumab (vascular endothelial growth factor receptor inhibitor for treatment of metastatic colorectal cancer), and gefitinib and cetuximab (EGF receptor inhibitor for treatment of lung and colorectal cancer) ${ }^{15-18}$ In addition to these, several drugs capable of inhibiting kinase activity through targeting their active site are currently in clinical trials. ${ }^{19}$ Although potent in inhibiting the activity of the kinase, this approach owing to the structural similarities in the active sites of the different kinases, especially the ATP binding site, results in reduced specificity and increased side effects. To overcome these deficiencies, efforts are ongoing to target other sites on the protein kinase critical for substrate engagement and phosphate transfer, such as the substrate-docking domain in MAP kinases. ${ }^{20}$ Targeting specific kinase-substrate interactions is also a potential strategy.
Volume 5 Issue 5 - 2017

\author{
Devanand Venkatasubbu G \\ Department of Nanotechnology, SRM University, India
}

\begin{abstract}
Correspondence: Devanand Venkatasubbu G, Department of Nanotechnology, SRM University, Kaatankulathur, Tamil Nadu, India,Email gdevanand@gmail.com
\end{abstract}

Received: April 16, 2017 | Published: May 31, 2017

However, such an approach would need an understanding of direct substrates of the particular protein kinase, structural details on their interaction, and availability of targetable structural features. Several recent technologies have accelerated the discovery of direct substrates of protein kinases. ${ }^{21}$ This information together with structural studies might pave way for more targeted inhibition of kinase-substrate pairs in future.

\section{Competitive and non-competitive peptide inhibitors}

The specificity of protein kinases for substrate phosphorylation typically depends on the primary amino acid sequences immediately flanking the site of phosphorylation, called the 'consensus sequence'. The different protein kinases differ in their preference for sequence specificity. This difference consensus sequence can be harnessed to develop specific inhibitory peptides that can act as a competitive inhibitor. A good example of a competitive kinase inhibitory peptide is peptide derived from the natural PKA inhibitor protein, PKI. ${ }^{22}$ PKI contains an RRNAL motif that resembles the consensus sequence of PKA (RRXS) ${ }^{23}$ However, the phosphoacceptor serine residue in PKA consensus site is replaced by alanine in the PKI sequence. ${ }^{24}$ Synthetic short peptides derived from the PKI sequence (5-22 residues) are effective inhibitors of PK. ${ }^{23}$ Another example is the peptide inhibitors derived from the unique recognition motif of GSK-3, S1XXXS2 (p), in which the second serine (S2) is pre-phosphorylated by another kinase. $^{24}$

\section{Targeting chaperones involved in protein kinase folding and translation}

Protein kinases can also be targeted indirectly using drugs that modify either their translation or folding. The heat shock protein Hsp90 is known to regulate the folding, stability and function of several proteins including protein kinases. ${ }^{25}$ Considering its impact on a wide range of functions, targeting Hsp90 is being thought of as a strategy that provides a means to simultaneously target multiple cancer promoting pathways. Most Hsp90 inhibitors developed to date are ATP nucleotide mimetics that block the essential ATPase activity of the chaperone, leading to degradation of protein kinases and other proteins that depend on Hsp90 for proper folding. Geldanamycin (a Hsp90 inhibitor) has been shown to retard the growth of ovarian 
cancer cells in xenograft mouse models, suggesting the potential and promise of this strategy. ${ }^{26}$

\section{Nanoparticle-mediated kinase targeting}

The potential of nanotechnology to make unprecedented and paradigm changing breakthrough in disease diagnosis and therapy has contributed to the immense interest and excitement in developing technologies using nanoparticles in drug delivery and medicine. Nanoparticles are usually in the size range of $60-200 \mathrm{~nm}$ and are capable of carrying multiple payloads for targeted transport, immune evasion and promote favorable drug release kinetics at the target site. ${ }^{27}$ The similarity in size of nanoparticles to cellular components such as cell surface receptors and signaling molecules enables them to interact and interfere with the activity of biological molecules efficiently. Nanoparticle-based drug delivery systems have enabled us to overcome several limitations of conventional drug delivery tools such as poor absorption and solubility, rapid metabolism and elimination, and bioavailability.

Although protein kinase has been successfully developed as a anti-cancer target, only about $5 \%$ of protein kinase inhibitors entering clinical trials reach marketing approval. ${ }^{27}$ The major stumbling block in translating these drugs from bench-to-bedside being drug related toxicity, which causes the shelving of an otherwise potent drug. The ability of nanotechnology-based approaches achieve selective delivery of drugs to the tumor leaving healthy cells untouched could help overcome this obstacle and significantly benefit discovery of targeted cancer therapeutics. There are several existing examples nanoparticles successfully being employed to enable selective targeting of protein kinase pathways in tumor cells. The use of selective MAPK specific inhibitor PD980059 chemically conjugated to an engineered hex dentate-PLGA polymer and was shown to inhibit the proliferation of melanoma and lung cancer cells in vitro and sensitize tumor cells to cisplatin chemotherapy in melanoma tumor bearing mice..$^{27,28}$ In another instance, encapsulating LY294002, a potent PI3K inhibitor into bio-degradable PLGA nanoparticle resulted in inhibition of downstream AKT phosphorylation ultimately resulting in inhibition of proliferation and induction of apoptosis of B16/F10 melanoma cells in vitro. ${ }^{29}$ Moreover, the nanoparticle-enabled targeting of the PI3K pathway also result inhibition of B16/F10 and MDA-MB-231 induced angiogenesis in a zebra fish tumor xenograft model. ${ }^{29}$ Also, gold and silver nanoparticles in the size range of $2-100 \mathrm{~nm}$, have been engineered to have their surface coated with Herceptin. ${ }^{27,30}$ These were then used to target ERBB2 receptors in human breast cancer SK-BR-3 cell lines..$^{30} \mathrm{~A}$ significant reduction in AKT and MAPK activation was observed followed by repressed expression of cyclin D $1 .{ }^{30}$ All these examples collectively show that nanoparticles have been successfully employed in achieving selective targeting of protein kinase pathways in cancer cells. However, this area is still new and largely unexplored. Nevertheless there is no denying the fact that nanotechnology-based approaches to deliver the inhibitors selectively to the tumor has the potential to revolutionize drug delivery and targeted disruption of signaling pathways driving human diseases such as cancer leading to translation of more drugs from bench-to-bedside.

\section{Acknowledgements}

None.

\section{Conflict of interest}

The author declares no conflict of interest.

\section{References}

1. Dhanasekaran N, Premkumar Reddy E. Signaling by dual specificity kinases. Oncogene. 1998;17(17):1447-14455.

2. Milanesi L, Petrillo M, Sepe L, et al. Systematic analysis of human kinase genes:a large number of genes and alternative splicing events result in functional and structural diversity. BMC Bioinformatics. 2005;6(Suppl 4):S20.

3. Manning G, Whyte DB, Martinez R, et al. The protein kinase complement of the human genome. Science. 2002;298(5600):1912-1934.

4. Padmanabhan A, Li X, Bieberich CJ. Protein kinase A regulates MYC protein through transcriptional and post-translational mechanisms in a catalytic subunit isoform-specific manner. $J$ Biol Chem. 2013;288(20):14158-14169.

5. Padmanabhan A, Gosc EB, Bieberich CJ. Stabilization of the prostatespecific tumor suppressor NKX3.1 by the oncogenic protein kinase Pim-1 in prostate cancer cells. J Cell Biochem. 2013;114(5):1050-1057.

6. Padmanabhan A, Rao V, De Marzo AM, et al. Regulating NKX3.1 stability and function: Post-translational modifications and structural determinants. Prostate. 2016;76(6):523-353.

7. Chisholm C, Achuth Padmanabhan, Bin Guan, et al. Protein Kinase CK2 Controls the Stability of Prostate Derived ETS Factor. The Open Cancer Journal. 2010;3:109-115.

8. Toughiri R, Padmanabhan A, Li X, et al. Discovering a new physiological substrate of Aurora-A kinase. Cancer Research. 2011;71(8):1619-1619.

9. Irby RB, Yeatman TJ. Role of Src expression and activation in human cancer. Oncogene. 2000;19(49):5636-5642.

10. Ganguly SS, Plattner R. Activation of abl family kinases in solid tumors. Genes Cancer. 2012;3(5-6):414-425.

11. Normanno N, De Luca A, Bianco C, et al. Epidermal growth factor receptor (EGFR) signaling in cancer. Gene. 2006;366(1):2-16.

12. Burotto M, Chiou VL, Lee JM, et al. The MAPK pathway across different malignancies: a new perspective. Cancer. 2014;120(22):3446-3456.

13. Fruman DA, Rommel C. PI3K and cancer: lessons, challenges and opportunities. Nat Rev Drug Discov. 2014;13(2):140-156.

14. Nagar B. c-Abl tyrosine kinase and inhibition by the cancer drug imatinib (Gleevec/STI-571). J Nutr. 2007;137(6):1518S-1523S

15. Hortobagyi GN. Trastuzumab in the treatment of breast cancer. $N$ Engl $J$ Med. 2005;353:1734-1736.

16. Liu TC, Jin X, Wang Y, et al. Role of epidermal growth factor receptor in lung cancer and targeted therapies. Am J Cancer Res. 2017;7(2):187202.

17. Lin Z, Yang Y, Huang Y, et al. Vascular endothelial growth factor receptor tyrosine kinase inhibitors versus bevacizumab in metastatic colorectal cancer: A systematic review and meta-analysis. Mol Clin Oncol. 2015;3(4):959-967.

18. Hudziak RM, Lewis GD, Winget M, et al. p185HER2 monoclonal antibody has antiproliferative effects in vitro and sensitizes human breast tumor cells to tumor necrosis factor. Mol Cell Biol. 1989;9(3):1165-7112.

19. Rask-Andersen M, Zhang J, Fabbro D, et al. Advances in kinase targeting: current clinical use and clinical trials. Trends Pharmacol Sci. 2014;35(11):604-620.

20. Padmanabhan A. Targeting Protein Kinase Substrate Docking in Cancers Journal of Glycobiology. 2014;3:e110.

21. Li X, Guan B, Srivastava MK, et al. The reverse in-gel kinase assay to profile physiological kinase substrates. Nat Methods. 2007;4(11):957962. 
22. Scott JD, Fischer EH, Takio K, et al. Amino acid sequence of the heatstable inhibitor of the cAMP-dependent protein kinase from rabbit skeletal muscle. Proc Natl Acad Sci USA. 1985;82(17):5732-5736.

23. Dalton GD, Dewey WL. Protein kinase inhibitor peptide (PKI): a family of endogenous neuropeptides that modulate neuronal cAMP-dependent protein kinase function. Neuropeptides. 2006;40(1):23-34.

24. Eldar-Finkelman H, Eisenstein M. Peptide inhibitors targeting protein kinases. Curr Pharm Des. 2009;15(21):2463-2470.

25. Zhao R, Houry WA. Hsp90:a chaperone for protein folding and gene regulation. Biochem Cell Biol. 2005;83(6):703-710.

26. Mo Q, Zhang Y, Jin X, et al. Geldanamycin, an inhibitor of Hsp90, increases paclitaxel-mediated toxicity in ovarian cancer cells through sustained activation of the p38/H2AX axis. Tumour Biol. 2016;37(11):14745-14755.
27. Basu S, ChaudhuriP, Sengupta S. Targeting oncogenic signaling pathways by exploiting nanotechnology. Cell Cycle. 2009;8(21):3480-3487.

28. Basu S. Nanoparticle-mediated targeting of MAPK signaling predisposes tumor to chemotherapy. Proc Natl Acad Sci U S A. 2009;106:7957-7961.

29. Harfouche R, Basu S, Soni S, et al. Nanoparticle-mediated targeting of phosphatidylinositol-3-kinase signaling inhibits angiogenesis. Angiogenesis. 2009;12(4):325-338.

30. Jiang W, Kim BY, Rutka JT, et al. Nanoparticle-mediated cellular response is size-dependent. Nat Nanotechnol. 2008;3:145-150. 